\title{
Article \\ Disinclusion of Palatally Impacted Canines with Surgical and Photobiomodulating Action of a Diode Laser: Case Series
}

\author{
Alessandra Impellizzeri ${ }^{1, *}$, Martina Horodynski ${ }^{1}$, Adriana De Stefano $\left.{ }^{2}{ }^{(}\right)$, Elisabetta Guercio-Monaco ${ }^{2}$,

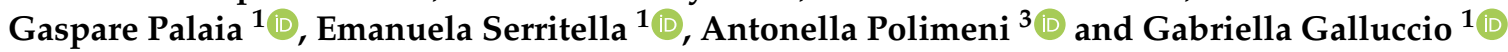 \\ 1 Department of Oral and Maxillofacial Sciences, "Sapienza" University of Rome, via Caserta 6, \\ 00161 Rome, Italy; Horodynski.1525899@studenti.uniroma1.it (M.H.); gaspare.palaia@uniroma1.it (G.P.); \\ emanuela.serritella@uniroma1.it (E.S.); gabriella.galluccio@uniroma1.it (G.G.) \\ 2 Department of Orthodontics, Faculty of Dentistry, University Central of Venezuela, Caracas 1050, Venezuela; \\ adriana.destefano@uniroma1.it (A.D.S.); Elisabetta.guercio@uniroma1.it (E.G.-M.) \\ 3 Post-Graduate School of Pediatric Dentistry, "Sapienza" University of Rome, via Caserta 6, 00161 Rome, Italy; \\ antonella.polimeni@uniroma1.it \\ * Correspondence: alessandra.impellizzeri@uniroma1.it
}

Citation: Impellizzeri, A.; Horodynski, M.; De Stefano, A.; Guercio-Monaco, E.; Palaia, G.; Serritella, E.; Polimeni, A.; Galluccio, G. Disinclusion of Palatally Impacted Canines with Surgical and

Photobiomodulating Action of a Diode Laser: Case Series. Appl. Sci. 2021, 11, 4869. https://doi.org/ 10.3390/app11114869

Academic Editors: Dorina Lauritano and Rosa Valletta

Received: 22 April 2021

Accepted: 24 May 2021

Published: 26 May 2021

Publisher's Note: MDPI stays neutral with regard to jurisdictional claims in published maps and institutional affiliations.

Copyright: (c) 2021 by the authors. Licensee MDPI, Basel, Switzerland. This article is an open access article distributed under the terms and conditions of the Creative Commons Attribution (CC BY) license (https:// creativecommons.org/licenses/by/ $4.0 /)$.

\begin{abstract}
Background: The permanent maxillary canine is the most impacted tooth after third molars There are many possible surgical techniques to approach impacted canines. The literature reports that high-intensity laser therapies (HILTs) can be used for opercolectomy of an impacted tooth. The aim of this study is to propose a new orthodontic-surgical approach using a laser for the disinclusion of palatally impacted canines. Methods: Nine patients presented maxillary primary canine persistence in the dental arch. Orthopanoramic X-ray and cone beam computed tomography (CBCT) showed the impaction of permanent maxillary canines. Surgical exposure was performed using a diode laser (Raffaello, DMT, Lissone, Italy, $980 \mathrm{~nm}+645 \mathrm{~nm}$ ). No orthodontic devices were applied for impacted tooth traction. Canine movement was monitored at 1, 8, 16 weeks post-surgery with photos and a CS3500 intraoral scanner (Carestream Dental, Atlanta USA) to evaluate their autonomous eruption. Results: No complications were observed. In all the cases, complete disimpaction of the treated canines was obtained in only four months. Conclusions: Impacted canine exposure with a diode laser has many advantages if compared with traditional surgery. The pre-orthodontic exposure and autonomous eruption of impacted canines provided simplified and predictable outcomes. The absence of traction and the reduced time for orthodontic treatment increased compliance during the orthodontic alignment.
\end{abstract}

Keywords: diode laser; ortho-surgical treatment; palatally impacted canines; disinclusion; no orthodontic traction; photobiomodulation

\section{Introduction}

Impacted teeth are often encountered during the diagnosis and treatment of malocclusions in adolescent orthodontic patients. After the third molars, the most commonly impacted tooth is the maxillary canine with an incidence of $0.9 \%$ to $2.2 \%$ [1-3], which varies depending on the ethnicity of the sample population.

Although the canine crown can be impacted either labially or palatally, it is more frequently positioned in the palate.

Of all patients who have impacted maxillary canines, $8 \%$ have bilateral impactions. They are more common in female than in male patients [4].

Over the years, numerous researchers have focused on identifying the cause of impacted maxillary canines. The etiology of palatally impacted canines has been suggested as polygenic and multifactorial. There are two main theories: the genetic theory and the guidance theory [5]. 
The therapeutic approach involves surgical exposure of the impacted tooth, followed by orthodontic traction to guide and align the tooth to the dental arch. For palatally impacted canines, various surgical approaches and various orthodontic traction techniques have been described in the literature [6]. There are two basic surgical methods for the exposure of a palatally impacted canine, the open and the closed technique. The open technique includes the surgical exposure of the crown by either complete removal of bone and soft tissue directly overlying the impacted canine and an orthodontic attachment is directly bonded on the canine in order to directly apply traction.

The closed technique, on the other side, involves raising a full mucoperiostal flap, exposing the canine crown, and bonding an attachment on it. Afterwards, the flap is repositioned, and orthodontic traction is applied after initial healing, until the canine erupts in the oral cavity and is subsequently guided to the dental arch $[5,7,8]$.

Nowadays, laser technology has a more important role in modern dentistry and, in the last two decades, there has been an increase in research studies on many laser applications.

The use of the laser offers several advantages when compared with conventional methods, including precision, minimal intraoperative hemorrhage, sterilization of the surgical area, healing with minimal scarring, and decreased post-operative pain and swelling [9].

The aim of this study is to show the effectiveness of laser technology for the exposure of the crowns of palatally impacted canines, using a diode laser device (Raffaello, DMT, Lissone, Italy, $980 \mathrm{~nm}+645 \mathrm{~nm}$ ), without orthodontic device application to traction it.

The hypothesis was that palatally impacted canines, after the laser surgery, will suffer a "spontaneous" eruption due to reactivation of the physiological eruption consequent to the laser stimulation and removal of the overlying tissue, without any orthodontic traction (traditional method).

The literature reports significant studies on photobiomodulation (PBM) demonstrating the biostimulation effect provided by some wavelengths of this type of laser [10-12]. In PBM, an activation of regenerative and healing processes can be observed, as a consequence of the ability of subcellular photoreceptors to react to visible red and near-infrared wavelengths. Stimulation of these receptors influences the electron transport chain, the respiratory chain, and oxidation, expressed as an increase in the cellular metabolic processes [12,13].

Furthermore, the literature reports that high-intensity laser therapies (HILTs), "surgical lasers" having a cutting action on the soft tissues [14-16], can also be used successfully for opercolectomy, with the removal of soft tissue that overlies the impacted tooth.

However, no study has evaluated the eruption-stimulating action when the laser is applied to expose the crown of impacted canines.

\section{Case Series}

The study was carried out on patients referred to the Orthodontics UOC of the Department of Odontostomatological and Maxillo-Facial Sciences of the "Sapienza" University of Rome. The period of recruitment of the patients was 6 months.

The inclusion criteria considered in the experimental study design were:

- $\quad$ Patients affected by palatally impacted canines.

- $\quad$ Age between 12 and 19 years.

- Patient considered reliable for follow-up.

- Understanding the protocol and able to give informed consent.

The exclusion criteria were:

- Non-cooperative patients.

- Previous orthodontic treatments.

- Inoperable patients.

- Vestibular impacted canines.

- $\quad$ Systemic pathologies.

- $\quad$ Subject to drug therapy. 
All the patients and their parents were informed with a written surgical consent about the treatment and its potential risks and benefits.

The final sample was constituted of 5 patients with one-sidely impacted canines and 4 patients with bilateral impactions, with a total of 13 impacted canines.

Orthopantomography (OPT) (Figure 1) and cone beam computed tomography (CBCT) (Figure 2) were requested for each patient at the beginning of therapy.

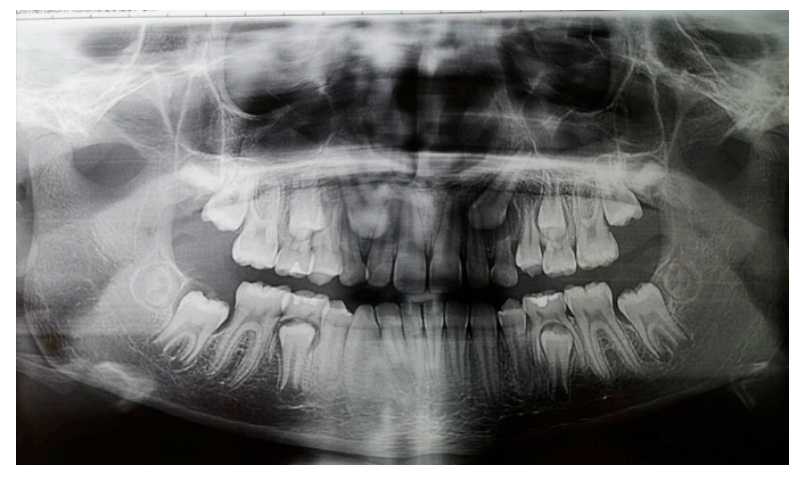

Figure 1. Panoramic radiograph detecting.

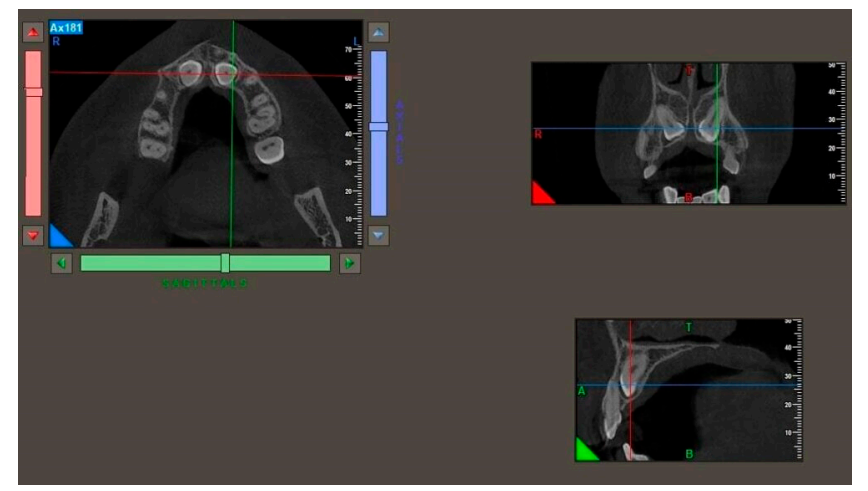

Figure 2. Bilaterally palatally impacted canines.

An evaluation of the prognosis of impacted canines was performed on OPT by two orthodontists, according to Ericson and Kurol [2]. The examination of the CBCT allowed us to evaluate the three-dimensional morphology of the impacted tooth, its location and inclination in the three planes of space, the depth and the type of inclusion, and the relationships with the other elements [17].

Nine of the 13 palatally impacted canines were covered by bone, while only three were covered by palatal mucosa. From the CBCT, it was possible to generate a threedimensional virtual model that could be superimposed and compared with the standard triangle language (STL) file obtained with an intraoral scanner during the monitoring.

In our clinical case studies, a diode laser was used (Raffaello ${ }^{\circledR}, \mathrm{DMT}$, Lissone, Italy, $980 \mathrm{~nm} / 645 \mathrm{~nm}$ ) with an optic fiber of $320 \mu \mathrm{m}$ and a power of $4 \mathrm{~W}$ in continuous mode (fluency: $4976 \mathrm{~J} / \mathrm{cm}^{2}$ ). This laser has high absorption in tissues pigmented with hemoglobin, melanin, and collagen chromophores. Therefore, the light energy from the diode is highly absorbed by the soft tissues and poorly absorbed by teeth and bone. The optical fiber delivery system touches the soft tissue and can be used for ablation, incision, and excision (cutting, ablation, curettage, coagulation, and hemostasis) [18,19]. In addition, the high frequency of the impulses allows an adequate thermal relaxation of the tissue, with minimal damage to the adjacent healthy areas, due to the spread of heat. Moreover, in most cases, there is no need to carry out any sutures. 
The surgical protocol includes a local anesthesia performed with a $3 \%$ solution of mepivacaine without adrenaline $(1.8 \mathrm{~mL})$. The operculum was realized with a diode laser (Figures 3 and 4 ) using the described parameters.

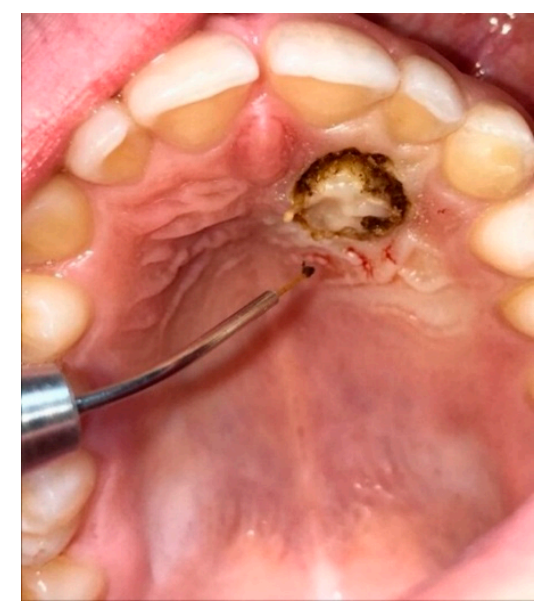

Figure 3. Laser surgery.

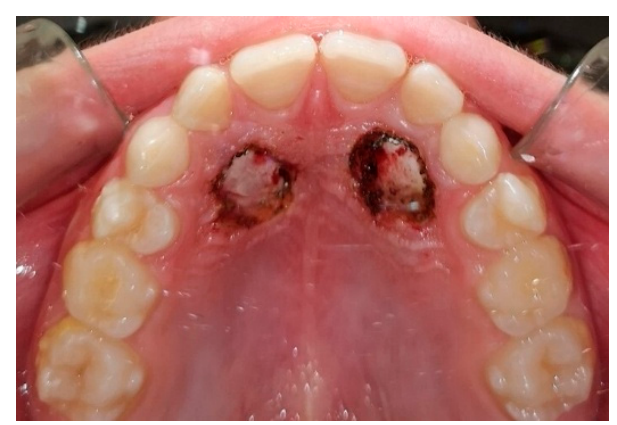

Figure 4. Laser surgery.

The fiber tip was used in contact with the tissue surface during the movement necessary to expose the crown of the impacted tooth. The palatal mucosa overlying the tooth was then detached with a Prichard periosteal elevator and removed by the laser. Afterwards, if the impacted tooth was covered by bone, an osteotomy was made through a contra-angle handpiece at low speed and under abundant irrigation with saline, with a rosette bur of ISO018 diameter (Maillefer ${ }^{\circledR}$, Ballaigues, Switzerland), using a tangential sliding movement to the bone tissue, to gradually remove it until the canine's surface was discovered without any damage.

Once the canine was clearly visible, the diode laser was passed over its surrounding surface to stimulate the periodontal ligament for the eruption movement activation, using a 600 micron fiber, at $0.1 \mathrm{~W}$, Ton $100 \mathrm{~ms}$, Toff $100 \mathrm{~ms}$, fluence $3.53 \mathrm{~J} / \mathrm{cm}^{2}$.

Finally, a periodontal dressing was applied (Coe-Pak ${ }^{\circledR}$, GC, Tokyo, Japan) to protect the treated area of the palate, for about 7 days, blocked by a suture point in Vicryl 3.0 (Ethicon ${ }^{\circledR}$ V311H 3/0 SH-1 70CM, J\&J Medical Devices, Somerville, MA, USA) (Figure 5). 


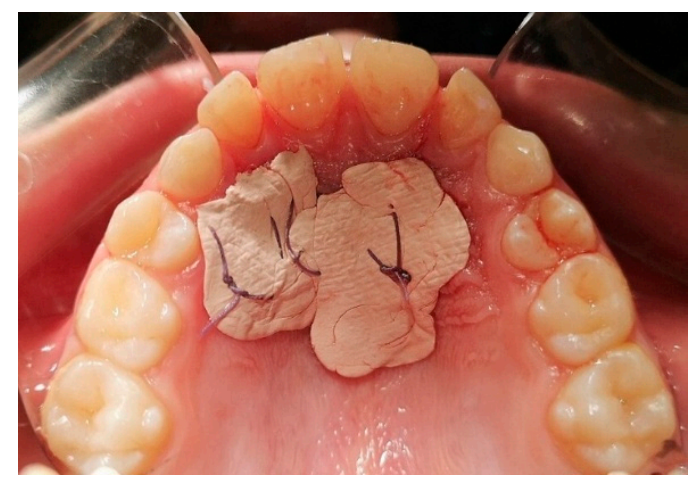

Figure 5. Periodontal dressing.

After 1 week, the periodontal dressing and the suture points were removed (Figure 6).

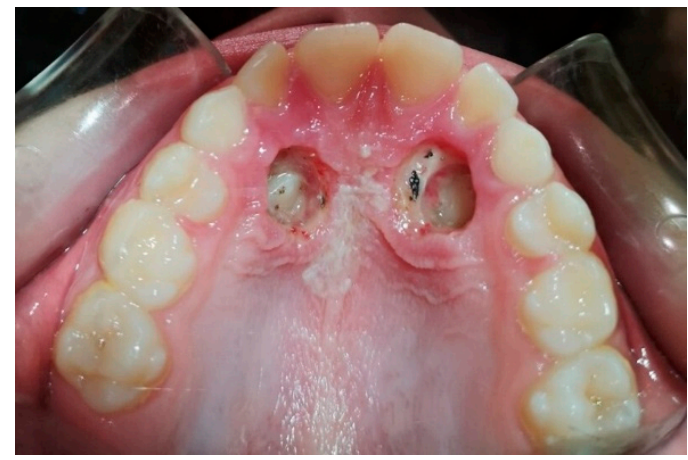

Figure 6. Intra-oral photo 1 week after surgery.

After surgery, patients underwent three check-ups at one week, 8 weeks, and 16 weeks (Figure 7) after the laser exposure, in order to evaluate and monitor, through photographic documentation [20] and digital color scans using the CS3500 intraoral scanner (CS3500 ${ }^{\circledR}$, Carestream Health, Atlanta, GA, USA), the eruption of the impacted tooth or the possible early closure of the mucosa and lack of appearance of the element in the palate.

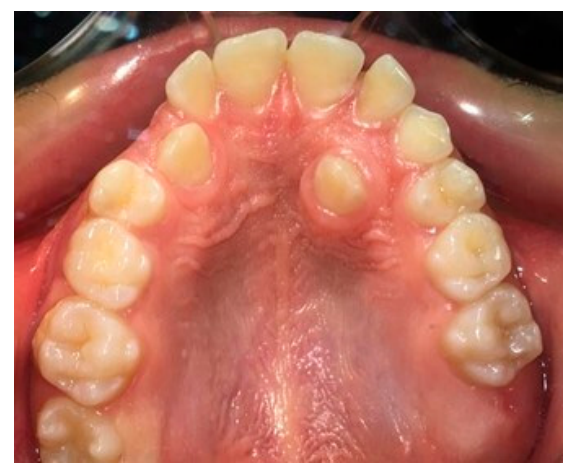

Figure 7. Intra-oral photo 16 weeks after surgery.

Moreover, clinical examination was carried out and a questionnaire about the occurrence of pain, swelling, bleeding, the need to take drugs, and reduction in the functionality of the area involved [15] was administered to the patients.

The 3D impressions (STL files) were then imported into the open-source software MeshLab $^{\circledR}$ (Visual Computing Lab, Pisa, Italy). After importing and superimposing the three scans files at one week and eight and sixteen weeks after surgery, the first operation was 3D data alignment. MeshLab provides a powerful tool for moving the different meshes into a common reference system, able to manage a large set of range-maps. 3D models often 
need to be re-oriented, or placed in a specific reference system; MeshLab provides a variety of features to manipulate the scale, positioning, and orientation of a 3D model, including basic transformation operations like translation/scaling/rotation, automatic re-centering and alignment to axis, geo-referencing with reference points, and interactive manipulators for rotation/translation/scaling. On the overlapping and aligned 3D models, through the "measuring tool", it was possible to millimetrically measure the distance between the visible cusp of the canine at one week after exposure and the cusp of the canine erupted on the palate at 8 and 16 weeks after laser surgery.

The "spontaneous" eruption was monitored through different digital technologies (CBCT, CS3500, Meshlab software, ISTI-CNR research center), from the time of the laser surgery to the crown eruption.

When the canines were erupted sufficiently into the palate, in an ideal period of 16 weeks, an orthodontic button was placed on the vestibular surface of the canines and the upper arch was bandaged with a self-ligating device [21,22]. Therefore, the tooth or teeth was or were gradually translated into the dental arch. No patients had any problems or intraoperative or post-operative complications.

\section{Results}

Through the superimposition (with the MeshLab software) of the 3D digital models obtained with the intraoral scanner CS3500, the millimetric values of spontaneous eruption of the palatally impacted canines treated by laser surgical exposure were obtained.

Table 1 contains all the total eruption data calculated for canine in T0-T2 (corresponding to eruption from 1 to 16 weeks) and the standard deviation (SD) for each canine considered in our study.

Table 1. Measurement of eruption and standard deviation of the 13 canines monitored at 16 weeks after the laser surgery.

\begin{tabular}{ccc}
\hline & Diode Laser Eruption Values $(\mathbf{m m})$ & SD \\
\hline 1 & 5.94 & 0.58 \\
2 & 3.75 & 1.61 \\
3 & 6.17 & 0.81 \\
4 & 3.12 & 2.24 \\
5 & 5.34 & 0.02 \\
6 & 4.18 & 1.18 \\
7 & 7.04 & 1.68 \\
8 & 6.9 & 1.54 \\
9 & 6.3 & 0.94 \\
10 & 5.91 & 0.55 \\
11 & 4.85 & 0.51 \\
12 & 5.38 & 0.02 \\
13 & 4.83 & 0.53 \\
\hline Mean & 5.36 & 0.94 \\
\hline
\end{tabular}

The mean eruption value, equal to $5.36 \mathrm{~mm}$, was therefore calculated, which corresponded to the amount of spontaneous movement of the canine on average over a 16-week period of monitoring after the laser surgery. The mean SD was $0.94 \mathrm{~mm}$.

Significant tooth movement was observed (Figure 8), and each canine responded positively to the laser surgery, without the need to repeat the procedure due to the closure of the operculum, despite the fact that nine of the 13 elements were in a deep bone inclusion. 


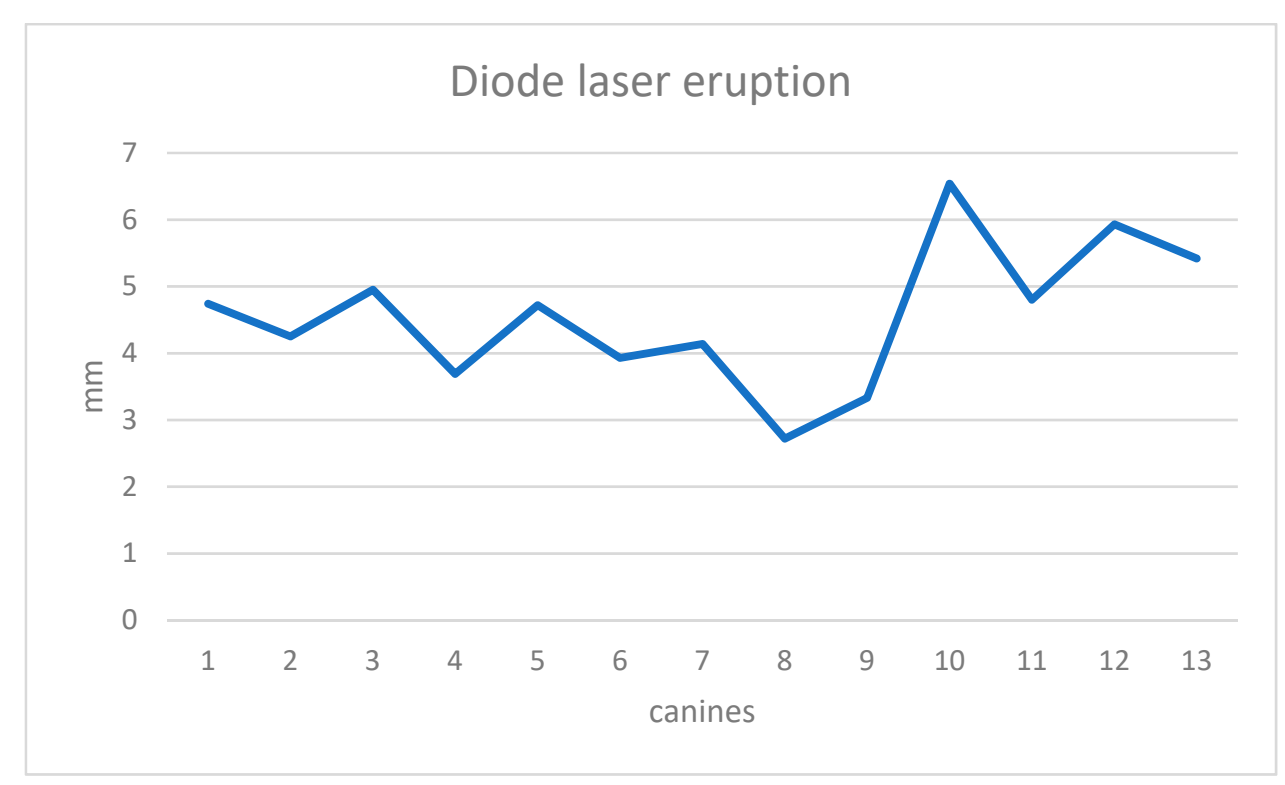

Figure 8. Values of eruption of the 13 canines at 16 weeks after laser surgery.

The speed of eruption was also considered for each canine. The eruption of the canines occurred with a mean speed of $0.045 \mathrm{~mm} /$ day. It was found that the mean speed in the first 8 weeks after surgery was greater than that in the remaining 8 weeks of monitoring. These data suggest that the periodontal activation of the impacted tooth could be greater in the first 2 months after the surgery, and it tended to slow down afterwards.

\section{Discussion}

The surgical-orthodontic canine disimpaction protocol used in this study differs from the conventional approach that involves immediate positioning of the bracket or button to exert the orthodontic force. This procedure can be difficult due to possible bleeding or to the reduced area of surgical exposure [23].

Moreover, in the conventional approach, the mechanical eruption of palatally impacted canines may lead to prolonged treatment, with an increased propensity for root resorptions and poor patient compliance [24,25]. Additionally, root resorption of teeth neighboring the impacted tooth is thought to be accelerated if mechanical eruption is undertaken $[25,26]$.

The surgical approach with the laser showed, compared to the traditional surgery, advantages already underlined in the literature: precision during the tissue incision, the need for a lower dose of local anesthesia, and the elimination of sutures with consequent reduction in infections and inflammatory processes. Furthermore, an antibacterial effect of the laser has been demonstrated [27], a property that improves post-operative admission and reduces the intake of antibiotics. No risks were found during laser application.

The literature reports numerous applications of lasers in oral surgery (HILT), for example, for opercolectomy in the surgical removal of soft tissue that overlies an impacted tooth [28]. However, the biostimulating action of HILT during the surgical exposure has not yet been demonstrated. With this study, we wanted to investigate it.

The application of PBM (LLLT) during orthodontic tooth movement has been widely investigated by different authors. Lasers acting in the "therapeutic window" (from about $600-1100 \mathrm{~nm}$ ) with an output energy of less than $500 \mathrm{~mW}$ are proven to have a biostimulating effect on the tissues without increasing the temperature of the treated region above normal body temperature. Therefore, they have the potential to accelerate tooth movement by means of influencing the remodeling of alveolar bone without unwanted impacts on the tooth and periodontium. Histologic investigations revealed that PBM during orthodontic tooth movement can profoundly affect cell-mediated alveolar bone remodeling [28].

Solmaz et al. used $635 \mathrm{~nm}$ diode laser irradiations at two different energy densities $\left(1\right.$ and $\left.3 \mathrm{~J} / \mathrm{cm}^{2}\right)$ to investigate the biostimulating effects on the healing of cutaneous skin 
wounds, in vivo, by means of bioimpedance measurements and histological examinations. Laser irradiations of both energy densities stimulated the wound healing process. In particular, laser irradiation of lower energy density had more evidence, especially for the first days of the healing process [29].

The purpose of our study was to propose a canine disinclusion protocol that would exploit the indisputable surgical qualities of the diode laser, which make it a very useful tool for these applications, and the biostimulating effect of a wavelength of $980 \mathrm{~nm}$, fully inserted in the therapeutic window. If, at the surgical level, we can say with certainty that the diode laser can represent a very useful tool because it offers countless surgical and post-operative advantages, the discussion relating to biomodulation deserves a separate mention and must be validated with an RCT and a control group. As for the spontaneous eruption of the canines without traction, it is true that some studies tell us that most canines could erupt with traditional methods, which, however, would lead to inconvenience for the patient during and after the operation (bleeding, discomfort due to the sutures [30]).

Our data suggest that the response of the dental element to the biostimulant action of the laser, applied to expose the crown, may be considered effective. The results obtained led us to hypothesize the effectiveness in photobiomodulation (PBM) on the dental use of a diode laser in the described surgical procedure, although it was performed on a small sample of patients.

The analysis of the speed of eruptions suggests that the stimulation effect of the laser could be more powerful in the first 8 weeks after the surgery, and it tended to slow down afterwards.

\section{Conclusions}

At the end of the evaluation period of 16 weeks, a significant spontaneous tooth movement was observed. The exposure of part of the dental crown, in all cases treated, can be considered valid to start the following phase of the fixed orthodontic therapy to bring the canine to its physiological position. Many advantages have emerged. Post-operative wound healing was rapid [29]. No patient complained of any pain or discomfort in the first post-operative check after a week. Furthermore, an increase in compliance of these young patients occurred, because they were subjected to a minimally invasive surgery that did not require the application of uncomfortable orthodontic devices on the palate.

The small sample is a limitation of the study, as well as the lack of a control scalpel group. Further clinical studies on a larger sample are required to obtain statistically significant data. In addition, future work will be focused on more detailed histological investigations, to evaluate, by means of tissue sampling and histological analysis, the cellular and tissue modifications generated by the laser action on an impacted canine.

Author Contributions: A.I. was a major contributor in writing the manuscript. M.H. analyzed and interpreted the patient data. A.D.S., E.G.-M., E.S. contributed analyze the data, G.P. helped to collect the data, A.P. and G.G. contributed to the review of the paper. All authors have read and agreed to the published version of the manuscript.

Funding: The authors received no financial support for the research, authorship, and/or publication of this article.

Institutional Review Board Statement: The study was conducted according to the guidelines of the Declaration of Helsinki and approved by the Ethics Committee of Sapienza University of Rome (\#4389) and was registered in the international public register. (Prot.N 0000847 22/06/18).

Informed Consent Statement: Written informed consent has been obtained from the patients and their parents to publish this paper.

Data Availability Statement: The datasets used and/or analyzed during the current study are available from the corresponding author on reasonable request.

Conflicts of Interest: The authors declare that they have no competing interests. 


\section{References}

1. Dachi, S.F.; Howell, F.V. A survey of 3874 routine full-mouth radiographs. A study of retained roots and teeth. Oral Surg. Oral Med. Oral Pathol. 1961, 14, 916-924. [CrossRef]

2. Ericson, S.; Kurol, J. Radiographic examination of ectopically erupting maxillary canines. Am. J. Orthod. Dentofac. Orthop. 1987, 91, 483-492. [CrossRef]

3. Bishara, S.E. Impacted maxillary canines: A review. Am. J. Orthod. Dentofac. Orthop. 1992, 101, 159-171. [CrossRef]

4. Becker, A.; Chaushu, S. Etiology of maxillary canine impaction: A review. Am. J. Orthod. Dentofac. Orthop. 2015, 148, 557-567. [CrossRef]

5. Cassina, C.; Papageorgiou, S.N.; Eliades, T. Open versus closed surgical exposure for permanent impacted canines: A systematic review and meta-analyses. Eur. J. Orthod. 2018, 40, 1-10. [CrossRef] [PubMed]

6. Cruz, R.M. Orthodontic traction of impacted canines: Concepts and clinical application. Dent. Press J. Orthod. 2019, 24, 74-87. [CrossRef]

7. Levin, M.P.; D'Amico, R.A. Flap design in exposing unerupted teeth. Am. J. Orthod. 1974, 65, 419-422. [CrossRef]

8. Becker, A.; Shpack, N.; Shteyer, A. Attachment bonding to impacted teeth at the time of surgical exposure. Eur. J. Orthod. 1996, 18, 457-463. [CrossRef]

9. Smith, P.W.; Arrastia, A.M.; Berns, M. Incision properties and thermal effects on three CO2 lasers in soft tissue. Oral Surg. Oral Med. Oral Pathol. 1995, 79, 685-691. [CrossRef]

10. Yassaei, S.; Fekrazad, R.; Shahraki, N. Effect of Low Level Laser Therapy on Orthodontic Tooth Movement: A Review Article. J. Dent. (Tehran Iran) 2013, 10, 264-272.

11. Ge, M.K.; He, W.L.; Chen, J.; Wen, C.; Yin, X.; Hu, Z.A.; Liu, Z.P.; Zou, S.J. Efficacy of low-level laser therapy for accelerating tooth movement during orthodontic treatment: A systematic review and meta-analysis. Lasers Med. Sci. 2015, 30, 1609-1618. [CrossRef] [PubMed]

12. Sonesson, M.; De Geer, E.; Subraian, J.; Petrén, S. Efficacy of low-level laser therapy in accelerating tooth movement, preventing relapse and managing acute pain during orthodontic treatment in humans: A systematic review. BMC Oral Health 2017, 17, 11. [CrossRef] [PubMed]

13. Johar, K. Low level laser therapy. In Fundamentals of Laser Dentistry; Jaypee Brothers Medical Publisher: New Delhi, India, 2011; pp. 128-132.

14. Impellizzeri, A.; Horodynski, M.; Fusco, R.; Palaia, G.; Polimeni, A.; Romeo, U.; Barbato, E.; Galluccio, G. Photobiomodulation therapy on orthodontic movement: Analysis of preliminary studies with a new protocol. Int. J. Environ. Res. Public Health 2020, 17, 3547. [CrossRef] [PubMed]

15. Impellizzeri, A.; Horodynski, M.; De Stefano, A.; Palaia, G.; Polimeni, A.; Romeo, U.; Guercio-Monaco, E.; Galluccio, G. CBCT and Intra-Oral Scanner: The Advantages of 3D Technologies in Orthodontic Treatment. Int. J. Environ. Res. Public Health 2020, $17,9428$. [CrossRef] [PubMed]

16. Impellizzeri, A.; Palaia, G.; Horodynski, M.; Pergolini, D.; Vernucci, R.A.; Romeo, U.; Galluccio, G. CO2 laser for surgical exposure of impacted palatally canines. J. Dent. CADM 2020, 88, 122-126. [CrossRef]

17. Impellizzeri, A.; Horodynski, M.; Serritella, E.; Romeo, U.; Barbato, E.; Galluccio, G. Three-dimensional evaluation of dental movement in Orthodontics. J. Dent. CADM 2020, 88, 182-190. [CrossRef]

18. Kravitz, N.D.; Kusnotob, B. Soft-tissue lasers in orthodontics: An overview. Am. J. Orthod. Dentofac. Orthop. 2008, 133, S110-S114. [CrossRef]

19. Boj, J.R.; Poirier, C.; Hernandez, M.; Espasa, E.; Espanya, A. Review: Laser Soft Tissue Treatments for Paediatric Dental Patients; European Archives of Paediatric Dentistry: Athens, Greece, 2011.

20. Impellizzeri, A.; Horodynski, M.; Barbato, E.; Polimeni, A.; Salah, P.; Galluccio, G. Dental Monitoring Application: It is a valid innovation in the Orthodontics Practice? Clin. Ter. 2020, 171, e260-e267.

21. Impellizzeri, A.; Putrino, A.; Zangrillo, C.; Barbato, E.; Galluccio, G. Efficiency of self-ligating vs conventional braces: Systematic review and meta-analysis. J. Dent. CADM 2019, 87,347-356. [CrossRef]

22. Putrino, A.; Impellizzeri, A.; Pavese, L.; Barbato, E.; Galluccio, G. Orthodontic treatment and third molars development: Longitudinal study on radiographs. J. Dent. CADM 2019, 87, 558-570. [CrossRef]

23. Caminiti, M.F.; Sandor, G.K.; Giambattistini, C.; Tompson, B. Outcomes of the surgical exposure, bonding and eruption of 82 impacted maxillary canines. J. Can. Dent. Assoc. 1988, 64, 572-4-576-9.

24. Padhraig, S.F.; Pratik, K.S.; DiBiase, A.T. How to ... mechanically erupt a palatal canine. J. Orthod. 2010, 37, $262-271$.

25. Segal, G.R.; Schiffman, P.H.; Tuncay, O.C. Meta-analysis of the treatment-related factors of external apical root resorption. Orthod. Craniofac. Res. 2004, 7, 71-78. [CrossRef]

26. Woloshyn, H.; Artun, J.; Kennedy, D.B.; Joondeph, D.R. Pulpal and periodontal reactions to orthodontic alignment of palatally impacted canines. Angle Orthod. 1994, 64, 257-264. [PubMed]

27. Verma, S.K.; Maheshwari, S.; Singh, R.K.; Chaudhari, P.K. Laser in dentistry: An innovative tool in modern dental practice. Natl. J. Maxillofac. Surg. 2012, 3, 124-132. [CrossRef]

28. Heidari, S.; Torkan, S. Laser Applications in Orthodontics. J. Lasers Med. Sci. 2013, 4, 151-158. [PubMed] 
29. Solmaz, H.; Dervisoglu, S.; Gulsoy, M.; Ulgen, Y. Laser biostimulation of wound healing: Bioimpedance measurements support histology. Lasers Med. Sci. 2016, 31, 1547-1554. [CrossRef]

30. Aldelaimi, T.N.; Khalil, A.A. Clinical Application of Diode Laser (980 nm) in Maxillofacial Surgical Procedures. J. Craniofac. Surg. 2015, 26, 1220-1223. [CrossRef] 